\author{
Marquette University \\ e-Publications@Marquette
}

Electrical and Computer Engineering Faculty

Research and Publications

Electrical and Computer Engineering,

Department of

\title{
Information-theoretic criterion for the performance of single- photon avalanche photodiodes
}

\author{
David A. Ramirez \\ University of Concepción \\ Majeed M. Hayat \\ Marquette University, majeed.hayat@marquette.edu \\ Sergio N. Torres \\ University of Concepcio'n \\ Bahaa E.A. Saleh \\ Boston University \\ Malvin Carl Teich \\ Boston University
}

Follow this and additional works at: https://epublications.marquette.edu/electric_fac

Part of the Computer Engineering Commons, and the Electrical and Computer Engineering Commons

\section{Recommended Citation}

Ramirez, David A.; Hayat, Majeed M.; Torres, Sergio N.; Saleh, Bahaa E.A.; and Teich, Malvin Carl, "Information-theoretic criterion for the performance of single-photon avalanche photodiodes" (2005).

Electrical and Computer Engineering Faculty Research and Publications. 539.

https://epublications.marquette.edu/electric_fac/539 
Marquette University

e-Publications@Marquette

\section{Electrical and Computer Engineering Faculty Research and Publications/College of Engineering}

This paper is NOT THE PUBLISHED VERSION; but the author's final, peer-reviewed manuscript. The published version may be accessed by following the link in the citation below.

IEEE Photonics Technology Letters, Vol. 17, No. 10 (2005): 2164-2166. DOI. This article is (C) Institute of Electrical and Electronic Engineers and permission has been granted for this version to appear in $\underline{\mathrm{e}}-$

Publications@Marquette. Institute of Electrical and Electronic Engineers does not grant permission for this article to be further copied/distributed or hosted elsewhere without the express permission from Institute of Electrical and Electronic Engineers.

\section{Information-theoretic criterion for the performance of single-photon avalanche photodiodes}

David A. Ramirez,

Department of Electrical Engineering, University of Concepción, Casilla 160-C, Concepción, Chile Majeed M. Hayat,

Department of Electrical and Computer Engineering, The University of New Mexico, Albuquerque, NM Sergio N. Torres, Department of Electrical Engineering, University of Concepción, Casilla 160-C, Concepción, Chile Bahaa E. A. Saleh, Department of Electrical and Computer Engineering, Boston University, Boston, MA Malvin C. Teich, Department of Electrical and Computer Engineering, Boston University, Boston, MA 


\section{Abstract}

A channel-capacity metric is introduced for assessing the performance of single-photon avalanche photodiodes (SPADs) when used as detectors in laser communication systems. This metric is employed to theoretically optimize, with respect to the device structure and operating voltage, the performance of SPADs with simple InP or In/sub 0.52/Al/sub 0.48/As-InP heterojunction multiplication regions. As the multiplication-region width increases, an increase is predicted in both the peak and the full-width at half-maximum of the channel capacity curve versus the normalized excess voltage. Calculations also show the existence of an optimal In/sub 0.52/Al/sub 0.48/As-InP heterojunction multiplication region that maximizes the peak channel capacity beyond that of $\ln P$.

\section{SECTION I. Introduction}

A commonly used performance metric for the performance of Geiger-mode avalanche photodiodes (APDs), also known as single-photon avalanche photodiodes (SPADs), is the single-photon quantum efficiency (SPQE), which is also referred to as the detection efficiency [1]. Another important metric is the dark-count probability $P_{d}$, which is one of the key factors affecting the SPQE. These metrics are well accepted and established for sensing applications, where the objective is to assess the capability of the SPAD in detecting and counting individual photons by examining the detection and false-alarm probabilities.

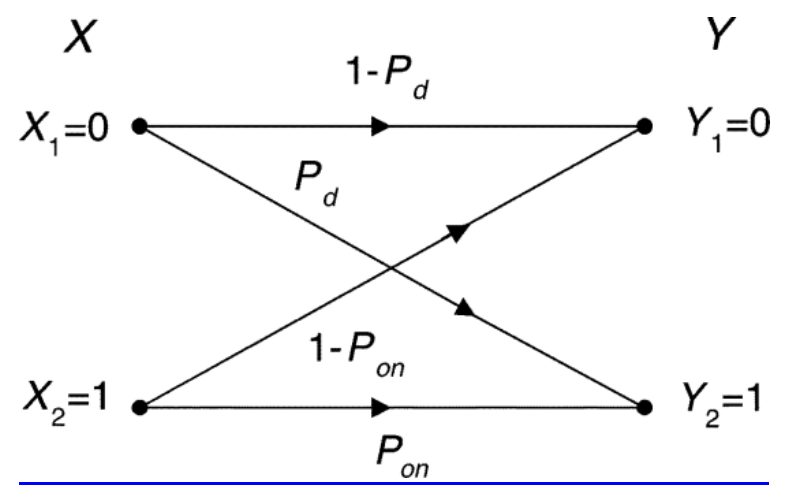

Fig. 1. SPAD communication channel.

Today, however, there are emerging applications in laser communications that require the use of SPADs as detectors [2], as they are used to determine the presence or absence of a photon in individual bits of optically transmitted binary data. For these applications, we can view the SPAD as a binary channel, in which case the channel capacity would be the natural metric for assessing the performance of the SPAD. In this context, the relevance of the channel capacity as a performance metric is clear: It represents the maximum rate of information we are allowed to transmit while maintaining the ability to reduce the bit-error probability to an arbitrarily small value through proper modulation and encoding for error correction [3]. Indeed, for certain deep-space communication systems, the current state of the art in single-photon detection is inadequate to achieve acceptable biterror probabilities [2]. Reliable operation of such systems would require effective error correction and modulation of the data to be transmitted. Thus, knowledge of the channel capacity of an SPAD is crucial since it provides the fundamental limit of the rate of information-transmission above which reliable operation is impossible. 
In this letter, we introduce the notion of channel capacity for single-photon detectors and utilize it to optimize the performance of SPADs with simple (InP) or heterojunction ( $\ln _{0.52} \mathrm{Al}_{0.48} \mathrm{As}-\mathrm{InP}$ ) multiplication regions. The optimization is performed over the operating voltage and the width of the multiplication region. It is shown theoretically that if the operational reverse-bias voltage of the SPAD is optimized with respect to the SPQE, the channel capacity achieved at that voltage is significantly below the maximum achievable value, which can be realized at a different voltage.

\section{SECTION II. Channel Capacity}

Consider the binary channel shown in Fig. 1 representing the input-output relationship of an SPAD. We call this channel the SPAD channel. The random variables $X \in\{0,1\}$ and $Y \in\{0,1\}$ represent, respectively, the possible input and output alphabets. In particular, $X=0$ represents the case when no optical power is present in a bit (zero signal) while $X=1$ corresponds to the case when an optical power is present. Similarly, $Y=0$ means that no avalanche breakdown has occurred during the detection period (bit) of the SPAD while $Y=1$ represents the occurrence of an avalanche breakdown. With these definitions, the transition probabilities of the SPAD channel can be identified with the total detection probability and the dark-count probability, which are described as follows. The total detection probability $P_{\text {on }}$ is the probability that either a dark carrier or a photon triggers an avalanche breakdown given that there is an optical pulse during the detection period. It is given by $P_{\text {on }}=1-e^{-\left(\eta P_{a} N_{o}+Q_{a} N_{d}\right)}$, where $P_{a}$ is the probability of an avalanche breakdown caused by a photocarrier that is injected into the multiplication region, $Q_{a}$ is the probability that a dark carrier (generated at a random location in the multiplication region) triggers an avalanche breakdown, and $N_{d}$ is the average number of dark carriers generated in the multiplication region during the detection time [4]. Here, the average number of photons per pulse is denoted by $N_{o}$, and $\eta$ is the quantum efficiency of the SPAD. The dark-count probability $P_{d}$ is given by $P_{d}=1-e^{-Q_{a} N_{d}}$ [1]. We now observe that sf $\operatorname{sf} \mathrm{P}\{Y=1 \mid X=0\}=$ $P_{d}, \mathrm{sf} \mathrm{P}\{Y=1 \mid X=1\}=P_{\text {on }}$, sf sf $\mathrm{P}\{Y=1 \mid X=0\}=P_{d}, \mathrm{sf} \mathrm{P}\{Y=1 \mid X=1\}=P_{\text {on, }}$ etc., as shown in Fig. 1. In this letter, the breakdown probabilities $P_{a}$ and $Q_{a}$ are calculated according to the dead-space multiplication theory [4], [5].

According to principles of digital communication, before defining the channel capacity we must define the mutual information of the SPAD channel, given by [3]

$$
\mathcal{J}=\sum_{j=0}^{1} \sum_{i=0}^{1} \operatorname{sfP}\{X=j, Y=i\} \frac{\operatorname{sf~}\{X=j \mid Y=i\}}{\operatorname{sf} \mathrm{P}\{Y=i\}}
$$

where the output-alphabet probabilities are given by sf sf $\mathrm{P}\{Y=0\}=\left(1-P_{d}\right) \pi_{0}+\left(1-P_{\text {on }}\right)(1-$ $\left.\pi_{0}\right)$, and $\pi_{0} \triangleq \operatorname{sf} \mathrm{P}\{X=0\}$ is the a priori input-symbol probability. Upon substitution, the mutual information for the channel shown in Fig. 1 reduces to 


$$
\begin{gathered}
\mathcal{J}=\pi_{0}\left(1-P_{d}\right) \log _{2}\left(\frac{1-P_{d}}{\left(1-P_{d}\right) \pi_{0}+\left(1-P_{\mathrm{on}}\right)\left(1-\pi_{0}\right)}\right) \\
+\pi_{0} P_{d} \log _{2}\left(\frac{P_{d}}{P_{d} \pi_{0}+P_{\mathrm{on}}\left(1-\pi_{0}\right)}\right) \\
+\left(1-\pi_{0}\right)\left(1-P_{\mathrm{on}}\right) \log _{2}\left(\frac{1-P_{\mathrm{on}}}{P_{d} \pi_{0}+P_{\mathrm{on}}\left(1-\pi_{0}\right)}\right) \\
+\left(1-\pi_{0}\right) P_{\mathrm{on}} \log _{2}\left(\frac{P_{\mathrm{on}}}{P_{d} \pi_{0}+P_{\mathrm{on}}\left(1-\pi_{0}\right)}\right)
\end{gathered}
$$

The channel capacity $\mathrm{C}$ with units of bits (or more generally, bits per symbol), can now be defined as the maximum of the mutual information $\mathcal{J}$ over all $0 \leq \pi_{0} \leq 1$, i.e., $C \triangleq \max _{0 \leq \pi_{0} \leq 1}$ J. It also follows that $0 \leq C \leq 1[3]$.

\section{SECTION III. Results}

In our calculations, we focus on the dark-carrier generation and breakdown only in the high electric field multiplication region of the SPAD. Band-to-band tunneling of carriers is considered as the dominant source of dark current in the multiplication layer [4], [6]. Throughout, we assume that the SPAD is operated in a gated mode, whereby it is dc-biased just below its breakdown voltage $V_{\mathrm{BR}}$ during the off-mode, and then pulse-biased above $V_{\mathrm{BR}}$ during the on-period. We assume a 2-ns on-pulse with repetition rate of $500 \mathrm{kHz}$ [6], $N_{o}=0.1$ photons, and $\eta=0.5$ [7].

The results of the calculations for an SPAD with a simple hole-injected InP multiplication region are shown in Fig. 2, which depicts the channel capacity as a function of the normalized excess voltage. (The normalized excess voltage is defined as $\Delta V / V_{\mathrm{BR}}$, where $\Delta V=V-V_{\mathrm{BR}}$ and $\mathrm{V}$ is the voltage across the multiplication region.) The peak channel capacity (shown by the symbol " $*$ ") and the corresponding reverse-bias voltage $V_{p}$ are shown for each width. Clearly, the peak channel capacity increases as the multiplication-region width increases. The key factor causing this performance enhancement is the reduction in the breakdown electric field (as the width is increased), which, in turn, leads to a reduction in tunneling dark carriers [6]. Another advantage of increasing the width of the multiplication region is reduced sensitivity to variation in the applied voltage. This is measured by the full-width at half-maximum (FWHM) of the channel capacity curve versus the normalized excess voltage; the FWHM increases as the multiplication-region width increases. 


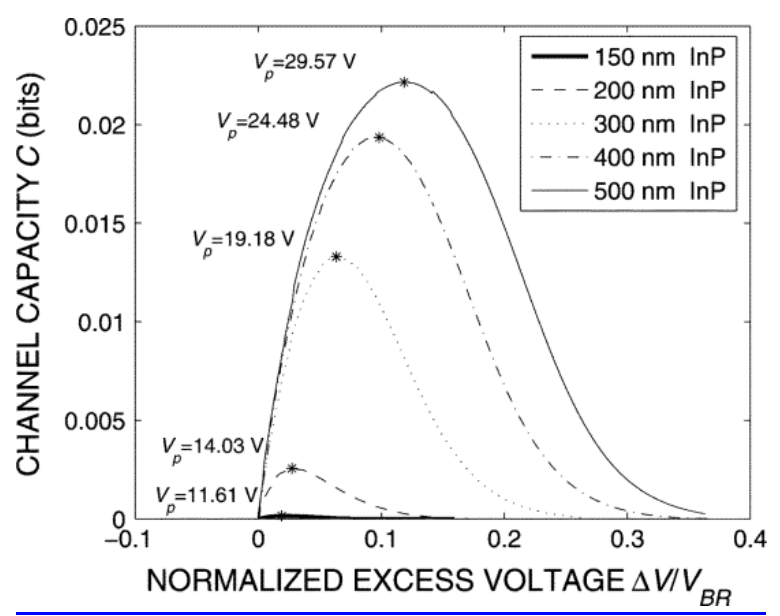

Fig. 2. Channel capacity corresponding to $\ln P$ multiplication region as function of the normalized excess voltage for each width. The reverse-bias voltage corresponding to the peak channel capacity $V_{p}$ is also shown for each width.

The trend in the channel capacity, as a function of the multiplication-region width, is similar to that of the SPQE observed in our earlier studies [4]. However, the voltage at which the channel capacity reaches its optimal value (peak channel capacity) is different from that corresponding to the optimal SPQE. For example, for a 500-nm multiplication region, the peak SPQE occurs at $32 \mathrm{~V}$ (according to the model reported in [4]) while the peak channel capacity occurs at $29.6 \mathrm{~V}$. Notably, if we reverse bias the SPAD at $32 \mathrm{~V}$, the channel capacity would be $40 \%$ below its peak value. Thus, it can be inferred that optimizations with respect to the SPQE and the channel capacity are not equivalent; SPAD optimization is, therefore, application (sensing versus communication) dependent.

We next consider an SPAD with an $\ln _{0.52} \mathrm{Al}_{0.48}$ As-InP heterojunction multiplication region, which has the same general structure of the heterostructure APDs considered for minimizing the excess noise factor [8]. For this type of SPAD, photogenerated holes are injected into the $\ln _{0.52} \mathrm{Al}_{0.48} \mathrm{As}$ layer, which is termed the energy-buildup layer. Our calculations indicate that for a fixed total width of the heterojunction multiplication region (consisting of both layers), the peak channel capacity depends on the fraction of the width occupied by the $\operatorname{In}_{0.52}$ Al $0_{0.48}$ As layer. The peak channel capacity (at the optimal applied voltage) starts at a minimum when the multiplication region is entirely made of $\operatorname{In}_{0.52} \mathrm{Al}_{0.48} \mathrm{As}$. As the fraction of the $\operatorname{In}_{0.52} \mathrm{Al}_{0.48}$ As-layer-width decreases, the peak channel capacity increases, reaching a maximum value, after which it decreases monotonically. This behavior is shown in Fig. 3 and it is attributable to the dead-space effect [4]. Thus, for a fixed total multiplication-region width, there is an optimal width of the $\ln _{0.52} \mathrm{Al}_{0.48}$ As layer that maximizes the peak channel capacity. For example, for total multiplication-region widths of 300 and $200 \mathrm{~nm}$, the optimal widths of the $\ln _{0.52} \mathrm{Al}_{0.48}$ As layer are 34 and $33 \mathrm{~nm}$, respectively. However, the channel capacity in this type of heterojunction is less sensitive to variations in the width of the $\ln _{0.52} \mathrm{Al}_{0.48}$ As layer compared to variations in the applied voltage. Thus, a small change in the width of the $\operatorname{In}_{0.52} A l_{0.48} A s$ layer (i.e., within $4 \mathrm{~nm}$ ) from its optimal value will not result in a significant change in the channel capacity. 


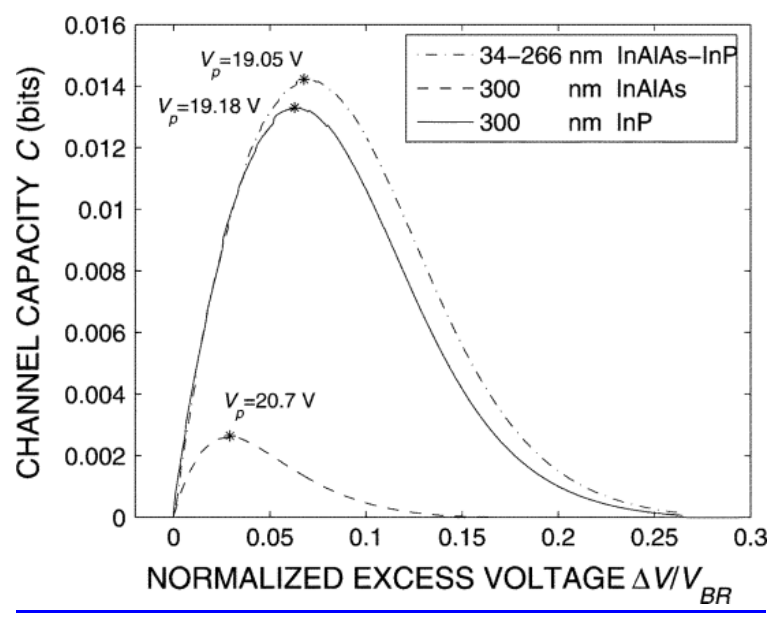

Fig. 3. Channel capacity corresponding to $\ln P, \operatorname{In}_{0.52} A l_{0.48} A s$, and optimal $\operatorname{In}_{0.52} A l_{0.48} A s-\operatorname{In} P$ multiplication regions of width $300 \mathrm{~nm}$. The reverse-bias voltage corresponding to the peak channel capacity $V_{p}$ is also shown for each multiplication region. The 34-266-nm heterostructure is the optimal structure among all other width combinations that have a total width of $300 \mathrm{~nm}$.

The peak channel capacities calculated are indeed very small (i.e., $\ll 1$ bits), which is a consequence of the ultraweak nature of the received signal (recall that $N_{o}=0.1$, corresponding to a subphoton level), which results in a high "miss" probability (e.g., sf sf $\mathrm{P}\{Y=0 \mid X=1\}=0.96$ in the case of a 300-nm InP SPAD operated at $19.2 \mathrm{~V}$.) If NoN_\{o\} is risen to 10 , for example, the peak-channel capacity increases to approximately 0.9 bits.

For a given total width of the multiplication region, the highest peak channel capacity occurs when the width of the $\operatorname{In}_{0.52} \mathrm{Al}_{0.48}$ As layer is between the hole' $s$ dead spaces in InP and $\operatorname{In}_{0.52} \mathrm{Al}_{0.48} \mathrm{As}$, at the breakdown field. (A similar behavior was shown earlier in the case of the SPQE [4].) This behavior can be explained in the context of the initial-energy effect [4], [9]: The high bandgap $\operatorname{In}_{0.52} \mathrm{Al}_{0.48} \mathrm{As}$ layer serves to energize (or heat) the injected holes before they enter the low-bandgap InP layer. If the width of the $\ln _{0.52} \mathrm{Al}_{0.48}$ As layer is just below the hole dead space in $\ln _{0.52} A l_{0.48} A s$, then the holes will energize without ionizing in the $\operatorname{In}_{0.52} \mathrm{Al}_{0.48}$ As layer. However, once they enter the InP layer, they require no dead space and may impact ionize immediately. This mechanism results in an enhancement in the breakdown probability, which in turn, reduces the electric field required for avalanche breakdown, thereby reducing tunneling dark carriers. Our analysis neglects phonon scattering, which may result in loss of energy of carriers as they are energized, thereby reducing the significance of the initial-energy effect.

Indeed, the calculated channel capacity of an optimized $\operatorname{In}_{0.52} \mathrm{Al}_{0.48} \mathrm{As}-\operatorname{InP}$ heterojunction is higher than that for a simple InP multiplication region of the same width, as shown in Fig. 3. The improvement in the peak channel capacity offered by the optimal In $0.52 \mathrm{Al}_{0.48} \mathrm{As}-\mathrm{InP}$ heterostructure, relative to the simple InP multiplication region, for the total multiplication-region widths of $100,200,300,400$, and $500 \mathrm{~nm}$ is $129 \%, 47 \%, 7 \%, 2 \%$, and $1 \%$, respectively. On the other hand, for the same range of widths, the improvement in the FWHM is $16 \%, 7 \%, 6 \%, 3 \%$, and $2 \%$, respectively. Note that the performance advantage is significant only in thin structures, which is due to the diminishing role played by the dead space in thick multiplication regions. 
A similar trend exits when considering the SPQE [4]; however, the optimal heterostructure that maximizes the channel capacity is different from that maximizing the SPQE. For example, when the total width is $300 \mathrm{~nm}$, the optimal SPQE-maximizing width of the $\mathrm{In}_{0.52} \mathrm{Al}_{0.48}$ As layer is $32 \mathrm{~nm}$, compared to $34 \mathrm{~nm}$ in the case of the channel-capacity optimization. Additionally, the applied voltage at which the SPQE peaks $(V=20.5 \mathrm{~V})$ is different from that at which channel capacity peaks $(\mathrm{V}=19 \mathrm{~V})$. If the channel-capacity-optimized heterostructure SPAD is operated at $V=20.5 \mathrm{~V}$, the channel capacity would be $58 \%$ below its maximum value (at $\mathrm{V}=19 \mathrm{~V}$ ).

\section{SECTION IV. Conclusion}

The channel capacity is the appropriate metric for the performance assessment and optimization of SPADs when used as detectors in a digital communication system. While both the channel capacity and the SPQE generally follow similar trends, device and operational-voltage optimizations based on these metrics are not equivalent; optimization with respect the wrong metric may result in a performance level that is well below the optimal performance.

\section{References}

1. Y. Kang, H. X. Lu, Y. H. Lo, D. S. Bethune, W. P. Risk, "Dark count probability and quantum efficiency of avalanche photodiodes for single-photon detection", Appl. Phys. Lett., vol. 83, pp. 2955-2957, 2003.

2. A. Biswas, W. H. Farr, "Detectors for ground-based reception of laser communications from Mars", 17th Annu. Meeting IEEE Lasers and Electro-Optics Society (LEOS), vol. 1, pp. 74-75, 2004.

3. J. G. Proakis, Digital Communications, New York:McGraw-Hill, 2001.

4. D. A. Ramirez, M. M. Hayat, G. Karve, J. C. Campbell, S. N. Torres, B. E. A. Saleh, M. C. Teich, "Generalized breakdown probabilities and detection efficiencies for single-photon avalanche photodiodes", IEEE J. Quantum Electron..

5. M. M. Hayat, Ü. Sakoĝlu (Sakoglu), O.-H. Kwon, S. Wang, J. C. Campbell, B. E. A. Saleh, M. C. Teich, "Breakdown probabilities for thin heterostructure avalanche photodiodes", IEEE J. Quantum Electron., vol. 39, no. 1, pp. 179-185, Jan. 2003.

6. G. Karve, S. Wang, F. Ma, X. Li, J. C. Campbell, " Origin of dark counts in In $\backslash \$\{0.53\} \backslash \$$ Ga $\backslash \$\{\{0.47\} \backslash \$$ As/In $\backslash \$\{\{0.52\} \backslash \$$ Al $\backslash\{\{0.48\} \backslash \$$ As avalanche photodiodes operated in Geiger mode ", Appl. Phys. Lett., vol. 86, pp. 63기-1-63미-3, 2005.

7. G. Karve, X. Zheng, X. Zhang, X. Li, S. Wang, F. Ma, A. Holmes, J. C. Campbell, G. S. Kinsey, J. C. Boisvert, T. D. Isshiki, R. Sudharsanan, D. S. Bethune, W. P. Risk, " Geiger mode operation of an In $\backslash \$\{0.53\} \backslash \$$ Ga $\backslash \$\left\{\{0.47\} \backslash \$\right.$ As-In $\backslash \$ \_\{0.52\} \backslash \$$ AI $\backslash \$\{0.48\} \backslash \$$ As avalanche photodiodes ", IEEE J. Quantum Electron., vol. 39, no. 10, pp. 1281-1286, Oct. 2003.

8. O.-H. Kwon, M. M. Hayat, S. Wang, J. C. Campbell, A. Holmes, B. E. A. Saleh, M. C. Teich, " Optimal excessnoise reduction in thin heterojunction $\mathrm{Al} \backslash \$ \_\{0.6\} \backslash \$ \mathrm{Ga} \backslash \$ \_\{0.4\} \backslash \$ \mathrm{As} / \mathrm{GaAs}$ avalanche photodiodes ", IEEE J. Quantum Electron., vol. 39, no. 10, pp. 1287-1296, Oct. 2003.

9. M. M. Hayat, O.-H. Kwon, S. Wang, J. C. Campbell, B. E. A. Saleh, M. C. Teich, "Boundary effects on multiplication noise in thin heterostructure avalanche photodiodes: Theory and experiment", IEEE Trans. Electron Devices, vol. 49, no. 12, pp. 2114-2123, Dec. 2002. 